\title{
OPENAIRE FÜR REPOSITORYMANAGERINNEN - WIE REPOSITORY MANAGERINNEN OPEN SCIENCE UNTERSTÜTZEN KÖNNEN
}

\section{von Olivia Kaiser und Gerda McNeill}

Zusammenfassung: Das EU-Projekt OpenAIRE unterstützt seit 2009 nun bereits im vierten Projektzyklus die Anstrengungen der Europäischen Kommission, wissenschaftliche Ergebnisse zugänglich, wiederverwendbar und offen zu gestalten. Mit der European Open Science Cloud (EOSC) strebt die EU bis 2022 die Schaffung einer Forschungsdatenplattform an, die Wissenschafterlnnen und Studierenden sowie der Öffentlichkeit zur Verfügung stehen soll. OpenAIRE Advance (2018-2020) ist in den Vorbereitungen zur EOSC mit anderen Projekten involviert und setzt sich hier zum Ziel, den Gedanken von Open Science einerseits zu verankern und andererseits die Infrastruktur zu verstetigen. Die Anbindung von institutionellen oder themenspezifischen Repositorien aus ganz Europa an das OpenAIRE-Portal stellt hierfür eine der Kernaufgaben dar. Schließlich ermöglicht diese eine leichterte Auffindbarkeit von Forschungsoutput und erhöht damit die Sichtbarkeit wissenschaftlicher Leistungen. Im vorliegenden Beitrag werden die Vorteile der Compliancy von institutionellen und disziplinspezifischen Repositorien im Kontext von Open Science diskutiert, es wird auf die technischen Voraussetzungen für eine solche eingegangen und zukünftige sich noch in Planung befindliche Services vorgestellt.

Schlagwörter: OpenAIRE; Open Science; Repositorium; Forschungsunterstützung

\section{OPENAIRE FOR REPOSITORY MANAGERS - HOW REPOSITORY MANAGERS CAN SUPPORT OPEN SCIENCE}

Abstract: OpenAIRE is an EU-project in it's fourth cycle with the aim to support the European Commission's goal to make science and research open, re-usable and accessbile since 2009. The EU targets to build the European Open Science Cloud (EOSC) as a research data plattform for researchers and students as well as the public till 2022. Along with other projects OpenAIRE Advance (2018-2020) is involved in the preperations towards the EOSC and has the mission to spread the idea of Open Science and strengthen the infrastructures needed. The compliancy of institutional and subject-specific repositories from all over Europe with the OpenAIRE-Portal is a key facilitator because it makes research output findable and rises the visibility. In this article the authors discuss the advantages of the compliancy and describe the technical requirements for doing so. 
Keywords: OpenAIRE; Open Science; Repository; Research Support

DOI: https://doi.org/10.31263/voebm.v72i2.2815

(c) Olivia Kaiser, Gerda McNeill

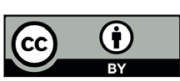

Dieses Werk ist lizenziert unter einer

Creative-Commons-Lizenz Namensnennung 4.0 International

\section{Inhalt}

1. Science. Set free: Das OpenAIRE Projekt

2. Compliancy - ist das kompliziert? Wie Repository ManagerInnen Open Science unterstützen können

3. Content Acquisition Policy von OpenAIRE

4. OpenAIRE goes EOSC

5. Resümee

\section{Science. Set free: Das OpenAIRE Projekt}

Das Motto „Science. Set free“ bringt es auf eine knappe Formel, um was es in OpenAIRE - Open Access Infrastructure for Research in Europe - geht. Seit 2009 unterstützt das paneuropäische Netzwerk das Open Access- und Open Science-Mandat der Europäischen Union. Als Meta-Forschungsinformationsplattform bietet OpenAIRE offenen Zugang zu Forschungsoutput und entwickelt laufend Services für verschiedene Zielgruppen im Forschungsprozess.

Forschende werden von Beginn des Forschungsvorhabens an dabei unterstützt, Open Science als Standard zu implementieren, wie etwa bei der Erstellung von Research Data Management Plänen, der Anwendung der FAIR (Findable, Accessable, Interoperabilable, Researchable)-Prinzipien beim Umgang mit Forschungsdaten oder auch beim Auffinden von geeigneten Repositorien.

Fördergebern, Forschungseinrichtungen und Forschungskoordinatorlnnen werden über das OpenAIRE-Portal Monitoring Services angeboten.

Um Openness gestalten zu können, werden Policies benötigt. OpenAIRE bringt sich hier auf europäischer und nationaler Ebene ein und nutzt die Expertise der verschiedenen Projektpartner und der Arbeit von anderen EU-geförderten Projekten, wie LEARN ${ }^{1}$ oder der Research Data Alliance $(\mathrm{RDA})^{2}$. 
OpenAIRE arbeitet eng mit Data und Content Providern zusammen. Dabei ist Interoperabilität das Stichwort, wenn es um die Schaffung und Weiterentwicklung der technischen Anbindung - die schließlich die Basis für die Verlinkung und Auffindbarkeit von Forschungsoutput ist - geht.

Mit Zenodo ${ }^{3}$ wurde in Zusammenarbeit mit CERN in Genf ein Repositorium geschaffen, das all jenen Projekten und Wissenschaftlerlnnen zur Verfügung steht, die über keine institutionellen oder fachspezifischen Repositorien an ihren Organisationen verfügen.

Es werden Schulungen zu den verschiedensten Themenschwerpunkten - z. B. in Form von Webinaren ${ }^{4}$ - angeboten, wobei eng mit dem Projekt FOSTER $^{5}$ in Bezug auf Forschungsdatenmanagement zusammen gearbeitet wird.

OpenAIRE setzt National Open Access Desks (kurz NOADs) ein, die sich als Open Science Helpdesks verstehen und EU-geförderte Projekte auf nationaler Ebene in Fragen rund um das Themenspektrum Open Science unterstützen.

Am Beginn von OpenAIRE stand im Jahr 2009 das Vorhaben, Forschungspublikationen, die peer-reviewed und Open Access sind, mit den zugehörigen Projektinformationen aus dem EU-Förderprogramm FP7 zu verlinken. Hierfür wurde 2010 das OpenAIRE-Portal ${ }^{6}$ eingerichtet, das sich aus Metadaten unterschiedlicher Quellen speist.

Seit 1. Jänner 2018 befindet sich OpenAIRE im vierten Projektzyklus und läuft bis Ende 2020 unter dem Titel OpenAIRE Advance. ${ }^{7}$ Der Fokus in Punkto Openness hat sich im Rahmen des Open Data Pilots im Rahmen des Vorgängerprojekts OpenAIRE Horizon2020 um Forschungsdaten erweitert. Im Sinne des Open Science-Gedankens werden heute die Metadaten von Forschungsdaten, projektrelevantem Forschungsoutput, Open Governmental Data und Open Educational Resources geharvestet, miteinander verlinkt und wo möglich, über das OpenAIRE-Portal zugänglich gemacht.

Das personelle und technische Netzwerk, das im Laufe der Jahre im Rahmen von OpenAIRE aufgebaut wurde, reicht über die EU-Mitgliedstaaten hinaus und arbeitet bereits mit assoziierten Projektpartnern aus der Türkei, Israel, Island, Norwegen, Serbien und Lateinamerika sowie Afrika zusammen. In Zukunft werden weitere Partnerschaften mit den USA und China angestrebt. Der Grundidee „Think global, act local“ folgend wurde ein Netzwerk von NOADs aufgebaut. Zumeist sind diese an Bibliotheken von Forschungsinstitutionen oder Universitäten angesiedelt, so dass sich Synergieeffekte ergeben. Die Erfahrung im Umgang mit Metadaten, Lizenzen, das Hosting eines Repositoriums sowie die Vernetzung der Bibliothe- 
karInnen innerhalb ihrer Einrichtungen und auf nationaler Ebene sind zentrale Bausteine zur Unterstützung OpenAIREs.

Die NOADs stehen als lokale Ansprechpartnerlnnen für Fragen rund um Open Access, Open Science und zur Erfüllung der Mandate im Rahmen von EU-Forschungsprojekten für alle Forschenden von nationalen Einrichtungen zur Verfügung.

Im Herbst 2018 entstand die gleichnamige Rechtspersönlichkeit OpenAIRE (legal entity), um Open Science auf europäischer Ebene bestmöglich und langfristig auch nach Projektende am 31. Dezember 2020 unterstützen zu können. Neben den ersten Unterzeichnern - Universität Minho (PT), Universität Göttingen, Athena Research Center und UNIT Norway - werden 2019 mehr als 10 weitere Einrichtungen Mitglieder der legal entity. ${ }^{8}$

\section{Compliancy - ist das kompliziert? Wie Repository ManagerInnen Open Science unterstützen können}

Immer mehr Fördergeber verlangen, dass projektbezogene Publikationen und in weiterer Folge ausgewählte Forschungsdaten Open Access zur Verfügung stehen.

Um das Open Access-Mandat im Rahmen der europäischen Forschungsförderung von FP7- und aktuell Horizon 2020 (H2020)-Projekten leichter erfüllen zu können, steht seit 2010 das OpenAIRE-Portal zur Verfügung. Publikationen, die durch das H2020-Programm gefördert werden, müssen Open Access veröffentlicht werden, wobei auch hier bei nachvollziehbarer Begründung eine opt-out-Möglichkeit gegeben ist. Die Europäische Kommission akzeptiert sowohl den Grünen Weg, bei dem das finale peer-reviewed Manuskript von den Wissenschafterlnnen in einem Open Access-Repositorium abgelegt wird, sowie auch den Goldenen Weg. Wenn die Gold Open Access-Variante gewählt wird, können etwaige Publikationsgebühren über das Projektbudget abgerechnet werden. ${ }^{9}$

OpenAIRE harvestet die Metadaten von Forschungsoutput in Repositorien oder Open Access-Journals. Die Metadaten stehen damit zusätzlich über das Portal zur Verfügung, sie sind teilweise bereits mit Projektinformationen sowie dem weiteren Projektoutput verlinkt und Nutzerlnnen werden über das Portal an die ursprüngliche Publikationsquelle weitergeleitet.

Das technische Team des Projekts OpenAIRE implementierte als Hilfestellung für Repository ManagerInnen die OpenAIRE-Guidelines zur Anbindung an das Portal und überarbeitet diese laufend. ${ }^{10}$ Es gibt derzeit drei 
Kategorien von Guidelines, die unterschiedliche Standards für die dementsprechenden Inhalte aufbereiten. Für Literatur-Repositorien wird Dublin Core, für Datenrepositorien das Datacite-Schema und für CRIS-Systeme CERIF-XML benutzt. Für die Einbindung von Software und Other Research Products in Repositorien wird aktuell an Guidelines gearbeitet. ${ }^{11}$

Damit ein Repositorium seine Inhalte auf der OpenAIRE-Plattform sichtbar machen kann, sind folgende Schritte nötig ${ }^{12}$ :

1. Erstellung eines Kontos auf: http://validator.openaire.eu

2. Registrierung des Repositoriums im Falle von Publikationen unter OpenDOAR ${ }^{13}$ bzw. im Falle von Forschungsdaten unter re3data ${ }^{14}$

3. Überprüfung mit Hilfe der OpenAIRE-Guidelines, ob das Repositorium die technischen Voraussetzungen erfüllt

4. Validierungstest über http://validator.openaire.eu

Bei Repositorien für Publikationen hat OpenAIRE eine Spezifizierung der unterschiedlichen Kompatibilitäts-Status durchgeführt. Maßgebend sind dabei die jeweilige Guideline-Version und der freigegebene Inhalt über die OAI Sets:

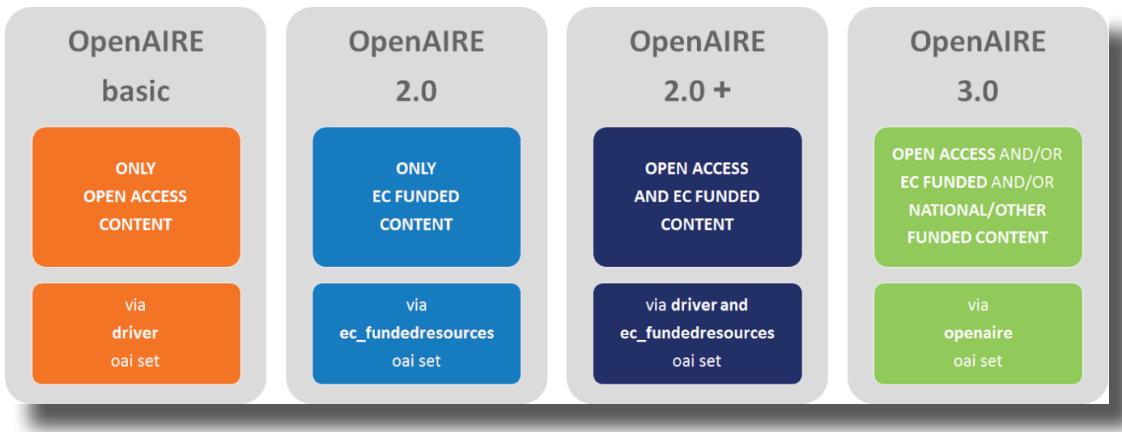

Abb. 1: OAI-Sets für OpenAIRE Compliancy ${ }^{15}$ bis zur Version 3.0

Seit den Guidelines 3.0 können zusätzlich zum Forschungsoutput, der im Rahmen von EU-geförderten Projekten entstanden ist, auch Forschungsergebnisse, die von anderen Fördergebern finanziert wurde, eingebunden werden. Als ein erfolgreiches Beispiel aus Österreich ist die auf Phaidra basierende FWF-E-Book-Library ${ }^{16}$ des Fonds zur Förderung der wissenschaftlichen Forschung (FWF) zu nennen. Der FWF verfügt über eine strikte Open Access-Policy für geförderte selbständige Publikationen. Diese müssen Open Access publiziert werden und über die E-Book-Library zur Verfügung stehen, deren Anbindung an OpenAIRE die Publikationen eben- 
so über das OpenAIRE-Portal auffındbar macht. Darüber hinaus sind über das Portal Artikel auffindbar, die im Rahmen eines FWF-geförderten Projekts entstanden sind. ${ }^{17}$ Das OpenAIRE-Portal unterstützt auf diesem Weg die Sichtbarkeit von Forschungsoutput, der im Rahmen von national geförderten Projekten entstanden ist.

Die Guidelines für Publikationsrepositorien beinhalten Informationen zu dem zu verwendenden Vokabular, wie Links zu Fördergebern und Forschungsoutput (wie etwa Forschungsdaten) einzufügen sind und wie Zugangsregelungen dokumentiert werden können. Das Update auf die Version 4.0, basierend auf Dublin Core und DataCite, ermöglicht die Unterstützung von Identifiern für AutorInnen, Organisationen und Fördergebern. ${ }^{18}$

Für Datenrepositorien enthalten die Guidelines zusätzlich das auf DataCite basierende XML-Schema. Im Fokus stehen jene Forschungsdaten, die mit einer Open Access-Publikation im OpenAIRE-Portal oder mit einem EU-geförderten Projekt in Verbindung stehen. Das OpenAIRE-Portal zeigt jene Datasets an, die als EU-Projekt identifizierbar sind und/oder mit einer Publikation im Portal verknüpft sind. Die Verknüpfung kann auch manuell vorgenommen werden.

Die Compliancy mit CRIS-Systemen ist über CERIF gegeben. Die ersten Guidelines wurden Anfang 2018 in der Community, insbesondere mit EuroCRIS ${ }^{19}$, gemeinsam diskutiert. An der Anbindung von PURE (Elsevier), dass von mehreren Institutionen in Österreich genutzt wird, wird intensiv gearbeitet.

Im Zuge der Weiterentwicklung von Services wird seit Ende 2018 mit dem Content Provider Dashboard eine one-stop-shop Lösung für Repository Managerlnnen angeboten. Damit ist es möglich, neben der Erstregistrierung und Validierung, eine Überprüfung des Harvestens vorzunehmen und statistische Daten abzufragen.

Vor kurzem wurde das OA Broker Service für Publikationsrepositorien in Form des Content Provider Dashboard veröffentlicht, der die Metadaten um etwa fehlenden Abstracts, IDs, Versionierungen, Zitierungen etc. anreichern wird. Repository Managerlnnen werden mittels Benachrichtigungen informiert werden.

Im Weiteren befindet sich das OpenAIRE Analytics Service in Entwicklung, das Nutzungsdaten der lokalen Repositorien sammelt und analysiert, um in weiterer Folge die article level metrics zu verbessern. Hierfür wird aktuell an Standards für die Sammlung von Nutzungsdaten ${ }^{20}$ gearbeitet sowie mit dem Einsatz von Big Data-Technologien experimentiert. ${ }^{21}$

Über die bestehenden Services informiert der OpenAIRE Service Catalogue. ${ }^{22}$ 


\section{Content Acquisition Policy von OpenAIRE}

Die technische Einbindung eines institutionellen oder fachlichen Repositoriums wird über die für den Forschungsoutput jeweils passenden Guidelines erklärt. Daneben stellt sich für Institutionen und Repositorien ManagerInnen die Frage, welche Inhalte durch die Anbindung geharvestet und im OpenAIRE-Portal sichtbar gemacht werden sollen. Die grundsätzliche Entscheidung liegt bei den Institutionen selbst.

Mit Oktober 2018 wurde eine neue Content Acquistion Policy von OpenAIRE veröffentlicht. ${ }^{23}$ Neben den Metadaten von Veröffentlichungen und Forschungsdaten werden in Zukunft auch die Metadaten von Software und „other research products“ geharvestet. Voraussetzung ist die Entsprechung der Guidelines der jeweiligen Quelle, wobei eine Open Access-Komponente nicht notwendig ist.

Um ein zukünftiges Monitoring für Fördergeber und Forschungsinstitutionen zu erleichtern, wird ein durch das Verlinken der verschiedenen Forschungsoutputs entstehende Graph herangezogen werden können.

\section{OpenAIRE goes EOSC}

Neben der kontinuierlichen Forcierung und Stärkung von Open Science ist es eine zentrale Aufgabe in OpenAIRE Advance, gemeinsam mit anderen Projekten wie EOSCpilot ${ }^{24}$, EOSC-Hub ${ }^{25}$ und elnfraCentral ${ }^{26}$ die European Open Science $\mathrm{Cloud}^{27}$ (EOSC) in ihrer Entstehung und Durchführung aktiv zu unterstützen. OpenAIRE und EOSC-Hub schlossen hierfür Ende April 2018 einen Kooperationsvertrag, in dem die Zusammenarbeit in den Bereichen Services, Strategie und Ressourcen und insbesondere Trainings ausgeführt wird. ${ }^{28}$ Die EOSC hat als europäische Initiative zum Ziel, eine virtuelle Forschungsumgebung aus bestehenden E-Infrastrukturen - Data und Content Provider - zu erstellen. Die Plattform ermöglicht das Speichern, Managen und Nutzen von Forschungsoutput an einem Ort. Insbesondere stehen als Zielgruppe Forschende und Studierende im Fokus, ebenso sollen auch Kleine und Mittlere Betriebe (KMU), die Industrie sowie die interessierte Öffentlichkeit von dem offenen Zugang zu Forschungsergebnissen profitieren. Am 23. November 2018 wurde das EOSC-Portal im Rahmen der österreichischen EU-Präsidentschaft an der Universitätsbibliothek Wien mit 350 internationalen Gästen feierlich eröffnet. Während des offiziellen Lounges des EOSC-Portals wurde die Vienna Declaration on the European Open Science Cloud ${ }^{29}$ von den anwesenden MinisterInnen aus Bulgarien, Rumänien und Österreich verlesen. 


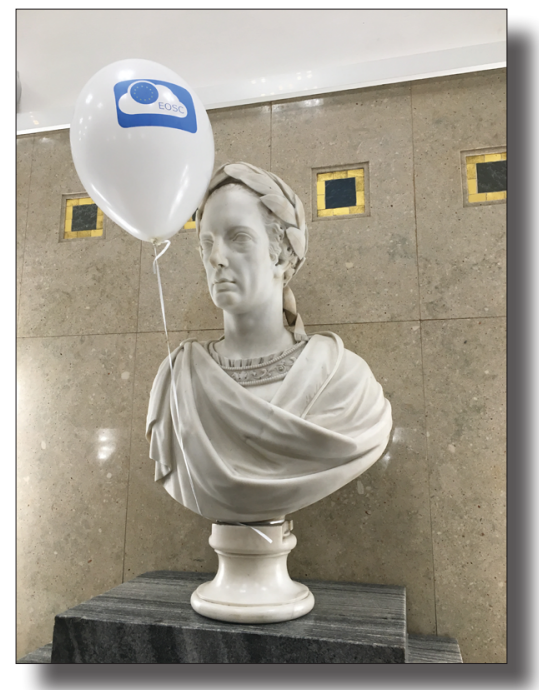

Abb. 2: EOSC Launch an der Universität Wien (ㄷ Olivia Kaiser)

OpenAIRE ist in der Governance-Struktur der EOSC vertreten und stellt ein Mitglied im Executive Board in der Person von Natalia Manola, Managing Director von OpenAIRE. ${ }^{30}$ OpenAIRE trägt in verschiedener Weise zur EOSC bei. Einerseits wird die Kultur von FAIR Data und Datenmanagement unterstützt, indem Fähigkeiten und Tools zu diesen Themen entwickelt, zur Verfügung gestellt und geschult werden. Andererseits ist geplant, dass alle Services von OpenAIRE über das EOSC-Portal zugänglich gemacht werden. Ebenso wird durch das große europaweite Netzwerk der NOADs dieser Paradigmenwechsel für die Wissenschaft in Europa auch auf nationaler Ebene gefördert und unterstützend begleitet.

\section{Resümee}

Offen zugängliche Repositorien werden immer essentieller und stellen einen Grundpfeiler für den freien Zugang zu Forschungsoutput dar. Dies bedeutet nicht nur eine Herausforderung für Wissenschafterlnnen, sondern auch für Forschungsinstitutionen und ihre IT-Abteilungen. Nicht zuletzt ist es selbstverständlich auch eine finanzielle Frage, ob institutionelle Repositorien betrieben werden können und wenn ja, welche Software dafür verwendet werden kann und wie diese auch technisch gewartet und weiter entwickelt werden kann. 
Abschließend ein Blick auf OpenAIRE aus der Praxis von Susanne Blumesberger, Repository Managerin von Phaidra an der Universitätsbibliothek Wien und welchen Beitrag Repository ManagerInnen zur Unterstützung von Open Science leisten: „Für Forschende ist die rasche und unkomplizierte Zugänglichkeit von Daten heute essentiell. Für aufwändige Recherchen bleibt im Forschungsprozess immer weniger Zeit. Daten, die auf unterschiedlichen Plattformen präsentiert werden, werden rascher aufgefunden und häufiger zitiert. Repositorien haben hier die Aufgabe, sich den Bedürfnissen der Wissenschafterlnnen anzupassen und die Forschung so effektiv wie möglich zu unterstützen. OpenAIRE wiederum bietet den RepositorienmanagerInnen Guidelines an, diese Aufgaben wahrzunehmen“.

\author{
Mag. ${ }^{a}$ Olivia Kaiser \\ Universität Wien, Bibliotheks- und Archivwesen \\ E-Mail: olivia.kaiser@univie.ac.at \\ Mag. ${ }^{a}$ Gerda McNeill \\ Universität Wien, Bibliotheks- und Archivwesen \\ E-Mail: gerda.mcneill@univie.ac.at
}

1 http://learn-rdm.eu/en/about/ (29.5.2019).

2 https://www.rd-alliance.org/ (29.5.2019) sowie RDA Austria Node; https://www.rd-alliance.org/groups/rda-austria (29.5.2019).

3 https://zenodo.org/ (29.5.2019)

4 https://www.openaire.eu/frontpage/webinars (29.5.2019).

5 https://www. fosteropenscience.eu/ (29.5.2019).

6 https://openaire.eu (29.5.2019).

7 OpenAIRE Advance ist ein Horizon 2020 Projekt. https://cordis.europa.eu/project/rcn/212961_en.html (11.2.2019) bzw. https://www. openaire.eu/openaire-advance-project (29.5.2019).

8 https://www.openaire.eu/openaire-organisation-in-the-making (29.5.2019).

9 http://ec.europa.eu/programmes/horizon2020/sites/horizon2020/ files/FactSheet_Open_Access.pdf (29.5.2019).

10 https://guidelines.openaire.eu/en/latest/ (29.5.2019).

11 Entwürfe der Guidelines können hier nachgelesen werden: https:// guidelines.openaire.eu/en/latest/ (29.5.2019).

12 https://www.openaire.eu/guides/ (29.5.2019).

13 http://v2.sherpa.ac.uk/opendoar/ (29.5.2019). 
14 https://re3data.org (29.5.2019).

15 OpenAIRE Compliancy - OAI Sets. https://www.openaire.eu/openaireguidelines-update-for-data-providers (04.07.2018).

16 https://e-book.fwf.ac.at/\#?page=1\&pagesize=10 (29.5.2019).

17 Mit Stand Mai 2019 befinden sich knapp 16.500 open access-Publikationen aus FWF-geförderten Mitteln im OpenAIRE-Portal. https:// explore.openaire.eu/search/find/publications?relfunder $=\% 22 \mathrm{fwf}$

::FWF||Austrian\%20Science\%20Fund\%20(FWF)||FWF\%22\&resul tbestaccessright $=\% 22$ Open\%20Access\%22 (29.5.2019).

18 OpenAIRE Guidelines for Literature Repository Managers v4. https:// openaire-guidelines-for-literature-repository-managers.readthedocs. io/en/v4.0.0/ (29.5.2019).

19 EuroCRIS ist eine internationaler Verein von Forschungsinformationsund dokumentationsexpertInnen. www.eurocris.org (29.5.2019).

20 Es besteht eine Zusammenarbeit mit der Initiative Institutional Repository Usage Statistics UK (IRUS) bezüglich eines Protokolls, dass in Kürze veröffentlicht werden soll.

21 An der Mitwirkung interessierte Repository Managerlnnen können ihr Interesse unterinfo@openaire.eu kundtun.

22 Siehe: OpenAIRE Service Catalogue. http://catalogue.openaire.eu/ search;quantity=9 (29.5.2019).

23 Siehe: OpenAIRE Content Acquisition Policies. https://zenodo.org/record/1446408\#.XGF7Z6BCdph (29.5.2019).

24 EOSCpilot stellt sich den technischen, wissenschaftlichen und kulturellen Herausforderungen der EOSC. https://eoscpilot.eu (29.5.2019).

25 EOSC-Hub baut sich zum zentralen Kontaktpunkt von Serviceprovider für Wissenschafterlnnen aus. http://eosc-hub.eu (29.5.2019).

26 elnfraCentral bemüht sich um die zielgruppenspezifische Bewerbung von bestehenden Infrastrukturen und hat hier insbesondere die Industrie im Blick. http://einfracentral.eu (29.5.2019).

27 EOSC Declaration vom 26. Oktober 2017. https://ec.europa.eu/research/openscience/index.cfm?pg=open-science-cloud (29.5.2019).

28 https: //www.eosc-hub.eu/collaborations/openaire-advance (29.5.2019).

29 https://eosc-launch.eu/fileadmin/user_upload/k_eosc_launch/ EOSC_Vienna_Declaration_2018.pdf(29.5.2019).

30 https://ec.europa.eu/info/news/results-call-applications-selectionmembers-expert-group-members-executive-board-eosc-2018-nov-23_en 